\title{
Genital tract function in men with Noonan syndrome
}

\author{
Madiha M Elsawi, John P Pryor, George Klufio, Carol Barnes, Michael A Patton
}

Department of Medical Genetics, St George's Hospital Medical School, Cranmer Terrace, London SW17 0RE, UK

M M Elsawi

M A Patton

King's College Hospital, Denmark Hill, London SE5 9PJ, UK

J P Pryor

G Klufio

\section{Lister Hospital,} Fertility and Endocrinology Centre, Chelsea SW1W 8RH, UK C Barnes

Correspondence to Dr Elsawi.

Received 12 November 1993 Revised version accepted for publication 18 February 1994

\begin{abstract}
In order to study the pattern of male fertility in Noonan syndrome, and its potential implications for genetic counselling, the genital tract function was studied in 11 adult males with Noonan syndrome. Bilateral testicular maldescent occurred in six. The mean testicular volume was 21 (SD 4) $\mathrm{ml}$. The stretched flaccid penile length was $11 \cdot 4$ (SD $1 \cdot 2) \mathrm{cm}$. Puberty was delayed in three. Four of the men had fathered children. The $L H$ and testosterone levels were essentially normal in all men, while the FSH levels were grossly raised in the group with testicular maldescent, with the exception of one man. Semen samples were obtained from five men, and azoospermia or oligozoospermia was present in four of them. Sexual function is not affected in men with Noonan syndrome, but the onset of sexual activity was delayed in men with late onset of puberty. Bilateral testicular maldescent appears to be the main factor contributing to impairment of fertility in men with Noonan syndrome.
\end{abstract}

(F Med Genet 1994;31:468-470)

The association of a characteristic facial appearance with pulmonary valvular stenosis and short stature was first reported by Noonan and Ehmke ${ }^{1}$ in 1963. Noonan syndrome may be the second commonest syndrome associated with congenital heart disease after Down's syndrome. The inheritance is autosomal dominant with a high spontaneous mutation rate, and the incidence may be between 1 in 1000 and 1 in 2000 live births. ${ }^{2}$ Major systems

Table 1 Clinical features in 11 adult males with Noonan syndrome

\begin{tabular}{|c|c|c|c|c|c|c|c|c|c|c|c|}
\hline & \multicolumn{11}{|c|}{ Patient } \\
\hline & 1 & 2 & 3 & 4 & 5 & 6 & 7 & 8 & 9 & 10 & 11 \\
\hline Small stature & + & + & + & + & - & + & - & + & + & + & + \\
\hline Characteristic facies & + & + & + & + & + & + & + & + & + & + & + \\
\hline Hypertelorism & - & + & + & + & + & + & + & + & - & + & + \\
\hline Downward slanting eyes & + & + & - & + & + & + & + & + & + & + & + \\
\hline Epicanthic folds & - & + & + & - & + & + & + & + & + & + & + \\
\hline Ptosis & + & + & + & - & - & + & + & + & + & + & + \\
\hline Low set ears & + & + & + & + & + & + & + & + & + & + & + \\
\hline Refractive errors & + & + & + & - & - & + & + & + & + & + & + \\
\hline Short neck & + & - & - & + & - & - & + & - & + & + & + \\
\hline Webbing & + & + & - & + & - & - & + & - & + & + & - \\
\hline Low posterior hair line & + & + & - & + & - & - & + & + & + & + & + \\
\hline Pectus excavatum & + & + & + & + & - & + & + & + & + & + & + \\
\hline Pectus carinatum & + & + & + & - & - & + & + & + & - & + & + \\
\hline Cubitus valgus & + & - & - & - & - & + & + & + & + & + & + \\
\hline Scoliosis/kyphosis. & + & + & - & - & - & - & - & - & + & - & + \\
\hline $\begin{array}{l}\text { Congenital heart disease } \\
\text { PS }\end{array}$ & & & & & & & & & & & \\
\hline PS & - & + & + & - & - & - & + & + & + & - & - \\
\hline Cardiomyopathy & + & - & - & - & - & - & - & - & + & - & - \\
\hline Other & - & - & - & - & - & - & - & + & - & + & + \\
\hline
\end{tabular}

affected include the cardiovascular, eye, and skeleton in addition to disorders of growth and development. Coagulation abnormalities have been recently shown in a large cohort of patients. ${ }^{3}$ Short stature is a common feature, although the nature of the growth failure is unknown, and up to three quarters of boys with this syndrome may have undescended testes.

It has been known that delayed puberty may occur in both sexes, but fertility does not seem to be affected in females. Previous studies in males have reported a variable pattern of pubertal development, ${ }^{4-6}$ but little is known about genital tract function. ${ }^{7}$ The aim of this paper was to study the genital tract function in men with Noonan syndrome.

\section{Methods}

In a previous study at St George's Hospital, ${ }^{8}$ 151 persons (83 males and 68 females) were diagnosed as having Noonan syndrome. The widest possible ascertainment was achieved through referrals from consultant paediatricians, paediatric cardiologists, and clinical geneticists in the UK, in addition to referrals from the Noonan Syndrome Society.

For the purpose of this work, ethical approval was given to recruit men 18 years or older who are of normal intelligence from the male cohort of the above mentioned study. ${ }^{8}$

Eleven of the 17 men who were approached by letter to join the study agreed and attended the hospital for a single visit. During this visit an andrological examination was performed which included measurement of the flaccid stretched penile length, and the assessment of the testis size by comparison with a rosary of ovoids. A seminal analysis was performed according to the WHO manual for semen analysis. ${ }^{9}$ A blood sample was obtained for hormonal assay and the serum was frozen and stored at $-20^{\circ} \mathrm{C}$ for later analysis. Plasma follicle stimulating hormone (FSH) and luteinising hormone (LH) were assayed using the Chelsea LH and FSH solid phase kit. The plasma testosterone levels were measured using an in house radioimmunoassay extraction.

The six males who refused to participate in the study had an age range of 19 to 34 years. Three of these men had undescended testes and another man had fathered four children (three of them had Noonan syndrome).

\section{Results}

All the men who attended were already known to have Noonan syndrome. Nine of them had short stature. The clinical features and labora- 
Table 2 Genital tract function in 11 adult males with Noonan syndrome

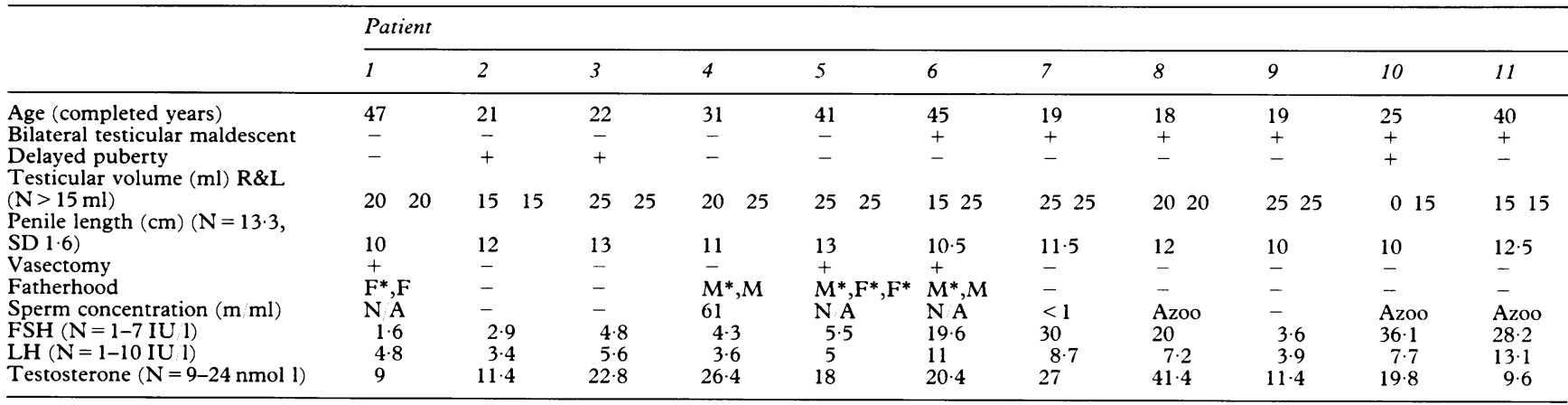

* = Affected with Noonan syndrome, $\mathrm{M}=$ Male, $\mathrm{F}=$ Female

$\mathrm{N} \mathrm{A}=$ Not available post vasectomy

tory findings are summarised in tables 1 and 2 . The mean age was 29.5 (SD 11) years (range 18-47). Testicular maldescent had occurred in six of the men and puberty was delayed in three (one with maldescent). The stretched penile length was $11.4(\mathrm{SD} 1.2) \mathrm{cm}$ (range 1013) and the mean testis volume was 21 (SD 4) $\mathrm{ml}$ (range 15-25). No patient reported any sexual difficulty, but the onset of sexual activity was delayed in men with late onset of puberty. The results of the hormone studies are shown in table 2. LH and testosterone levels were essentially normal in all patients, but the FSH levels were grossly raised in all men with testicular maldescent with the exception of one man who had normal sized testes. Four of the men had married (Nos 1, 4, 5, and 6), and had fathered children. Noonan syndrome occurred in their offspring confirming paternity. Three of them had undergone a vasectomy, and the fourth was able to produce a normal semen sample. Only one of these fathers (No 6) belongs to the group with testicular maldescent. He had normal sized testes, but a raised FSH level. The other four men with history of maldescent had azoospermia (3) or severe oligozoospermia (1), with raised plasma levels of FSH. It should be noted that two single men aged 21 and 22 with a history of delayed puberty were unable to produce a sample of semen at the time of their visit.

Of the original sample of 17 men contacted, nine had undescended testes and only one of them $(11 \%)$ fathered children. Four of the remaining eight men with normal descent had children.

\section{Discussion}

There has been a paucity of published reports dealing with the genital function in males with NS. ${ }^{111}$ Some of these were isolated case reports which looked at the hormonal profile of the patient or studied his testicular function. ${ }^{412}$

Theintz and Savage 5 reported five prepubertal boys with NS who had all undergone orchidopexy in early childhood. They found them all to have delayed skeletal maturation and pubertal development with an exaggerated LH response. The possible mechanism suggested is Leydig cell dysfunction. Saez et al ${ }^{13}$ were unable to find evidence for this in their study. They estimated plasma testosterone, dehydroepiandosterone and its sulphate in two males with NS before and after administration of human chorionic gonadotrophin (HCG). Their age was 10 and 17 years and both had normal testicular descent. Both patients had a sharp rise in plasma testosterone after stimulation with HCG.

Sinisi $e t a l^{14}$ studied four men with Noonan syndrome. Their age ranged from 19 to 23 years and two of them were mentally retarded with cryptorchidism. He found that the two with normal testicular descent had normal levels of FSH and LH. One of them had normal semen analysis. In contrast the two men with testicular maldescent had high levels of $\mathrm{LH}$, and one had high $\mathrm{FSH}$, and the only semen test showed azoospermia.

Kumanov $^{15}$ in 1985 studied four Noonan males aged 19 to 27 , none of whom had mental retardation. He mainly described their hormonal profile. One patient was normally virilised and had normal sexual function. Seminal analysis was only available in this patient and was within normal limits. Two patients had prepubertal testicular size, while the fourth had bilateral undescended testes. High levels of TRH and prolactin were found in all of them.

Okuyama et $a l^{16}$ studied the gonadal function in seven prepubertal cryptorchid boys with NS and found abnormalities of testicular function. Their age ranged from 5 to 16 years. Five of them had mild mental retardation. They all had LHRH and HCG stimulation and testicular biopsy. The histology in all testes showed interstitial fibrosis and four of seven had abnormalities of seminiferous tubules. Two of the seven had good response to hormonal stimulation. Testicular volume was reduced in six of seven, and one case showed early pubertal changes. This is not surprising as this patient was the oldest of the group.

In the present study we found no evidence of any Leydig cell deficiency, even in the men with testicular maldescent. Six of 11 men had a history of testicular maldescent and these patients had poor quality semen associated with a high level of plasma FSH, suggesting a failure of spermatogenesis. In contrast, men with normal descent had normal FSH levels and tended to be fertile. Penile length tended to be shorter than average, but no patient had a 
micropenis (average $-2.5 \mathrm{SD}$ ), nor did any man report any sexual problems related to penile length.

It has been shown that pubertal development is delayed in both sexes in Noonan syndrome. The mean age (SD) of menarche in 20 postpubertal women with Noonan syndrome was $14.6(1.17)$ years. ${ }^{8}$ Fertility does not appear to be affected in females. Most familial cases that have been reported have shown an overrepresentation of affected mothers. In our previous study, we had 16 familial cases, and in 12 of them the mother was the affected parent. The decreased paternal transmission is likely to reflect the impairment of fertility in males. In the clinical study of Char et al ${ }^{17}$ of 45 cases of Noonan syndrome there were five familial cases, and in three of them this was because of mother to daughter transmission. Nora et al ${ }^{18}$ described three families and in all three the condition was transmitted through the mother.

Maldescent is recognised to be an adverse factor on male fertility, and this is the case in men without Noonan syndrome. Chilvers et $a l,{ }^{19}$ in a review of published reports, found that $75 \%$ of men had azoospermia or oligozoospermia following orchidopexy for bilateral undescended testes.

In conclusion, it appears that the main factor leading to impairment of fertility in men with Noonan syndrome is the presence of bilateral cryptorchidism. In this study the mean agé at orchidopexy was 5.5 years. It remains to be seen whether earlier surgery would improve the outcome. In the presence of normally descended testes, men with Noonan syndrome are likely to be fertile.

We would like to thank the Lewis Family Charitable Trust and the Noonan Syndrome Society for funding and support of this research. We would also like to thank the staff of the Fertility and Endocrinology Centre at the Lister Hospital for their help with the study.
1 Noonan JA, Ehmke DA. Associated non cardiac malformations in children with congenital heart disease $\mathcal{J}$ Pediatr 1963;63:468-70

2 Nora JJ, Nora AH, Sinha AK, Spangler RD, Lubs HA. The Ullrich-Noonan syndrome (Turner phenotype). $\mathrm{Am}$ f Dis Child 1974;127:48-55.

3 Sharland M, Patton MA, Talbot S, Chitolie A, Bevan DH. Coagulation-factor deficiencies and abnormal bleeding in Noonan's syndrome. Lancet 1992;339:19-21.

4 Nistal M, Paniagua R, Pallardo LF. Testicular biopsy and hormonal study in a male with Noonan's syndrome. Andrologia 1983;15:415-25.

5 Theintz G, Savage MO. Growth and pubertal developmen in five boys with Noonan's syndrome. Arch Dis Child 1982;57:13-17.

6 Ranke MB, Heidemann P, Knupfer C, Enders H, Schmaltz AA, Bierich JR. Noonan syndrome: growth and clinical manifestations in 144 cases. Eur $\mathcal{F}$ Pediatr 1988;148:220 7.

7 Anonymous. Editorial. Noonan's syndrome. Lance 1992;340:22-3.

8 Sharland M, Burch M, McKenna WM, Patton MA. A clinical study of Noonan syndrome. Arch Dis Child 1992;67:178-83.

9 WHO. Laboratory manual for the examination of human semen and semen-cervical mucus interaction. Cambridge Cambridge University Press, 1987.

10 Ciovirnache $M$, Florea I, Popa M, Ionescu V, Popescu H Morphotypic aspects in the cryptorchidic child. Endocrinologie 1987;25:45-53.

11 Redman JF. Noonan's syndrome and cryptorchidism. Urol 1973;109:909-11.

12 Kauschansky A, Eilam N, Elian E. LR-RH and HCG studies in a Turner phenotype male (Noonan's syndrome). A case report. Helv Paediatr Acta 1977;32:23740.

13 Saez JM, Morera AM, Bertrand J. Testicular endocrine function in males with Noonan's syndrome. Lancet
1969;ii:1078-9.

14 Sinisi AA, Criscuolo T, Maresca F, et al. Endocrine profile in Noonan's syndrome. Minerva Endocrinol 1987;12:13-

15 Kumanov P. Elevated basal levels and exaggerated responses to thyrotrophin-releasing hormone of prolactin and thyrotrophin in Turner-Noonan-syndrome. Andrologia 1985;17:395-9.

16 Okuyama A, Nishimoto N, Yoshioka T, et al. Gonada findings in cryptorchid boys with Noonan's phenotype. Eur Urol 1981;7:274-7.

17 Char F, Rodriguez-Fernandez HL, Scott CI, Borgaonkar DS, Bell BB, Rowe RD. The Noonan syndrome - a clinical study of forty five cases. Birth Defects 1972;8:11018.

18 Nora JJ, Sinha AK. Inheritance of the Turner phenotype. Birth Defects 1969; V(5):29-35.

19 Chilvers C, Dudley NE, Gough MH, Jackson MB, Pike MC. Undescended testis: the effect of treatment on subsequent risk of subfertility and malignancy. $\mathcal{F}$ Pediatr Surg 1986;21:691-6. 